\title{
A role of ultrasonic frequency and power on oil mobilization in underground petroleum reservoirs
}

\author{
Hossein Hamidi • Roozbeh Rafati • \\ Radzuan Bin Junin • Mohammad A. Manan
}

Received: 25 April 2011/ Accepted: 9 January 2012/Published online: 20 January 2012

(C) The Author(s) 2012. This article is published with open access at Springerlink.com

\begin{abstract}
In oil industry, the reduction of oil production is of major concern as world's necessity for oil increases. Therefore, developing and applying new techniques to mobilize residual oil left in the reservoir is important. Ultrasound technique is one of the unconventional methods to increase the productivity of oil wells, but in spite of many laboratory experiments on oil mobilization under ultrasound in porous media the precise mechanisms are weakly understood. Therefore, it is vital to perform basic experiments to achieve a good knowledge and deep insight into the mechanisms. To recognize the reason why ultrasound is able to mobilize residual oil left in pores, one must remember why oil droplet is trapped. Residual oil is left in pores because of insisting capillary forces. So, in this paper a mechanism related to capillary forces are developed to clarify the effect of ultrasound on mobilization of residual oil in porous media. In addition, some experiments are conducted with a $2 \mathrm{D}$ glass micro-model in which the mobilization of oil is observed using a digital microscope and camera. It was concluded from the result of experiments that the oil mobilization was proportional to ultrasound power and frequency.
\end{abstract}

H. Hamidi $(\bowtie) \cdot$ R. Rafati · R. B. Junin · M. A. Manan Faculty of Petroleum and Renewable Energy Engineering, Universiti Teknologi Malaysia, Johor Bahru, Malaysia

e-mail: hossein_hamidi_59@yahoo.com

R. Rafati

e-mail: roozbeh.rafati@yahoo.com

R. B. Junin

e-mail: radzuan@petroleum.utm.my

M. A. Manan

e-mail: m-amanan@petroleum.utm.my
Keywords Ultrasonic - Power - Frequency · Mobilization · Oil

\section{Introduction}

After primary and secondary recovery of an oil field, using some conventional-enhanced oil recovery methods such as chemical flooding (surfactant flooding), polymer flooding, gas flooding, steam flooding for improving oil recovery are definitely required. However, these methods are expensive to use, need a wide range of a surface apparatus, generate dangerous environmental results, and have technical limitations. Therefore, some unconventional methods have been recommended with lower application risk. One of these unconventional methods is the application of ultrasound for oil reservoir stimulation. To clarify the effects of ultrasonic waves on oil recovery many laboratory experiments and field observations have been conducted during the past 50 years.

The interest in recovery of oil from oil reservoirs under vibration dates back to 1950 s when increased oil production was observed because of cultural noise and earthquakes. The viability of this method to increase oil recovery was first shown by Duhon and Campbell (1965). Chen (1969) and Fairbanks and Chen (1971) showed the effect of ultrasonic waves with the frequency of about $20 \mathrm{kHz}$ and power of $10 \mathrm{~W} / \mathrm{cm}^{2}$ on the oil and water flow through porous sandstone and through a capillary. They concluded that, ultrasound enhanced the percolation rate in the case of a porous medium. They also showed that this increase is only slightly due to ultrasonic heating.

Simkin and Surguchev (1991) and Simkin (1993) pointed out that oil droplet size was increased after 
applying ultrasonic waves. This increase was attributed to coalescence of oil droplets because of Bjerknes forces under ultrasound.

Kostrov and Wooden (2001) presented some possible oil recovery mechanisms as the following: (1) dislodging the oil droplet from the pore wall into the flow of fluid, (2) coalescence of two or more the oil droplets (oil films surrounding the formation grains) into the big one having higher mobility. Their results showed an increase in oil production to about $40 \%$ of total oil production.

Amro et al. (2007) conducted some experiments on core samples to show the effect of ultrasonic waves on oil mobilization. They concluded that oil displacement increased after applying ultrasonic waves and relative permeability changed. Furthermore, they concluded that applying ultrasound at residual oil saturation led to higher oil recovery compared to original oil in place.

Beresnev et al. (2005) explained a capillary-related mechanism to describe the mobilization of oil droplet inside porous media after applying ultrasonic waves. They conducted this study at pore scale on a non-wet oil droplet which was trapped at the entry of a pore in a channel, as showed in Fig. 1. As seen, the radius of the left meniscus is greater than the right one $\left(R_{\text {left }}>R_{\text {right }}\right)$. Therefore, an inequality of capillary pressure $(\Delta P)$ is generated inside the oil droplet that resisting the external pressure. As the oil droplet move more forward to the throat, the inequality of capillary pressure increases more. When the oil droplet is trapped, the pressure gradient $(\Delta P)$ inside it equals to the external pressure gradient $\left(\Delta P_{\mathrm{s}}\right)$. To move the droplets through the throat, $\Delta P_{\mathrm{s}}$ must be increased (more than $\Delta P$ ). In this case, the droplet starts to move. This additional pressure can be created by ultrasonic waves $\left(P_{\mathrm{U}}\right)$. It means that when $\Delta P_{\mathrm{s}}+P_{\mathrm{U}}>\Delta P$ then the oil droplet can pass through the throat. In addition, the force generated by ultrasonic wave increases with the increase of ultrasound power.

Based on the above literature, it can be predicted that the oil mobilization enhances with increasing ultrasound power. So, in this study to prove the claim, a comprehensive series of macro-model (Core sample) and micro-model experiments were done to show the effect of ultrasonic

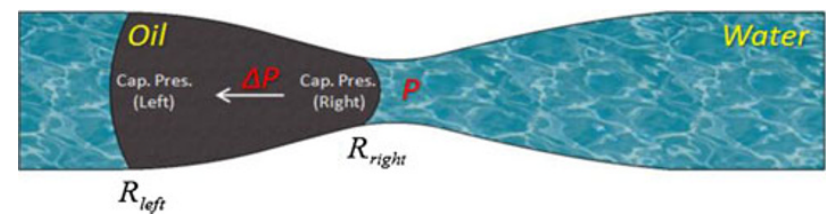

Fig. 1 A trapped oil under a $\Delta P$ (pressure gradient). The droplet radii are shown as $R_{\text {right }}$ and $R_{\text {left }}$. The term, Cap. Pres. refers to capillary pressure waves on oil recovery rate and oil mobilization for different medium wettability (oil wet and water wet), and oil viscosities $(15,100$, and $400 \mathrm{cp})$. In addition, the main parameters of ultrasound sources such as frequency (25 and $40 \mathrm{kHz})$ and power $(1-500 \mathrm{~W})$ were altered.

\section{Experimental setup and procedure}

\section{Macro-model experiments}

For macro-model experiments, co-current (all sides open to flow) Berea sandstone cores were used with the dimension of $6 \times 3 \mathrm{~cm}(L \times D)$. After saturating of the cores by oil in the vacuum, they were put inside of an imbibition cell filled up with brine. Then, for ultrasonic tests, the cells were placed inside an ultrasonic bath (ultrasonic cleaner) filled up with de-aerated water. We used two ultrasonic generators to produce the vibrational energy at frequencies of 25 and $40 \mathrm{kHz}$ and power of 250 and $500 \mathrm{~W}$, respectively. The schematic diagram of the imbibition experiment apparatus is shown in Fig. 2. The mineral oils used in experiments had various viscosities like 15, 100, and $400 \mathrm{cp}$. In some of the experiments for investigating the effect of different ultrasonic frequencies on oil recovery, we used the minimum energy output $(1 \mathrm{~W})$. The experimental runs are shown in Table 1.

\section{Micro-model experiments}

According to Fig. 3, after preparation and fabrication of the 2D glass micro-model, they were connected to the valves to which control the flow in and out of the model. The dimension of micro-model was about $60 \times 60 \mathrm{~mm}$ (Fig. 4). The pore and throat diameters were 740 and $280 \mu \mathrm{m}$, respectively. The unit was immersed into a bath which was filled with water to make a suitable surrounding for propagation of ultrasonic waves.

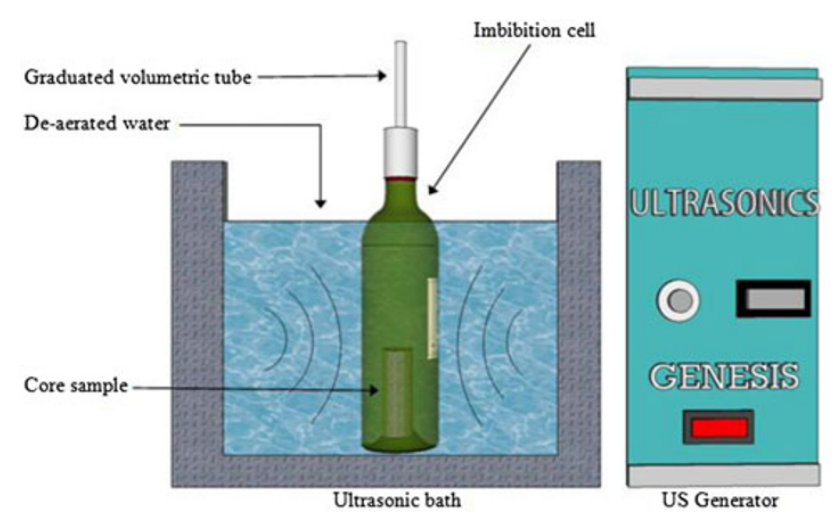

Fig. 2 Experimental setup for imbibition experiments 
Table 1 Experimental runs performed in this study

\begin{tabular}{llrrl}
\hline No. & Wettability & Viscosity (cp) & Power (W) & $\begin{array}{l}\text { Frequency } \\
(\mathrm{kHz})\end{array}$ \\
\hline 1 & Water wet & 400 & 500 & 25 \\
2 & Water wet & 400 & 250 & 25 \\
3 & Water wet & 400 & 0 & 25 \\
4 & Water wet & 100 & 500 & 25 \\
5 & Water wet & 100 & 250 & 25 \\
6 & Water wet & 100 & 0 & 25 \\
7 & Water wet & 15 & 500 & 25 \\
8 & Water wet & 15 & 250 & 25 \\
9 & Water wet & 15 & 0 & 25 \\
10 & Oil wet & 100 & 250 & 25 \\
11 & Oil wet & 100 & 0 & 25 \\
12 & Oil wet & 15 & 250 & 25 \\
13 & Oil wet & 15 & 0 & 25 \\
14 & Water wet & 100 & 1 & 40 \\
15 & Water wet & 100 & 1 & 25 \\
16 & Water wet & 15 & 1 & 40 \\
17 & Water wet & 15 & 1 & 25 \\
\hline
\end{tabular}

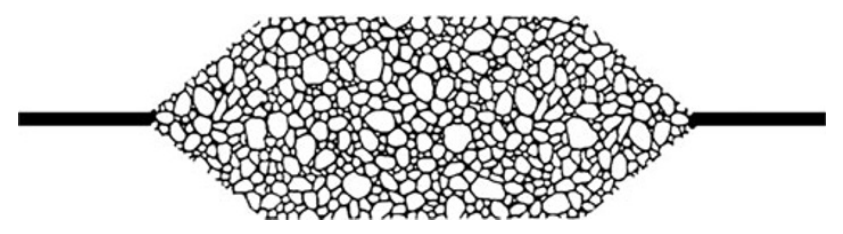

Fig. 3 Schematic of the micro-model
To run tests, at first, brine (dyed deep blue) with salinity concentration of $\mathrm{NaCl}=0.35 \mathrm{wt}(\%), \mathrm{MgCl}_{2}=0.25 \mathrm{wt}(\%)$, and $\mathrm{CaCl}_{2}=0.10 \mathrm{wt}(\%)$ was injected by a KD Scientific syringe pump into the micro-model to saturate the micro-model. Then, for several hours, micro-model was left intact. Afterward, the oil (dyed red) was injected at $1.0 \mathrm{ml} / \mathrm{h}$ to move the initial brine. The oil properties are shown in Table 2. The injection was continued until reaching a point in which only the oil was produced at the port. This point could be recognized using microscope and camera at the production port and throughout the micromodel. After recognizing this point the oil injection could be finished. In this stage, a condition of connate water saturation was provided throughout the micro-model. Then brine (dyed pale blue) with the salinity concentration equal to surfactant solution salinity concentration was injected into the micro-models at the injection rate of $0.018 \mathrm{ml} / \mathrm{h}$. It must be noted that, during the injection of brine, ultrasonic waves were also applied. Ultrasonic generator that was placed at the top of the micro-model generated these ultrasonic waves. A stereo microscope, Carl Zeiss Brand, Model Stemi SV 11 was focused at the pore scale. The camera mounted on this microscope monitored the displacement of the oil by water and sent the images to a PC for analyses. For calculating pores and throats area that occupied by the oil, the Image Analyzer Software was used. In these series of experiments, the ultrasound power and frequency for each experiment were different. The experimental runs are shown in Table 3.

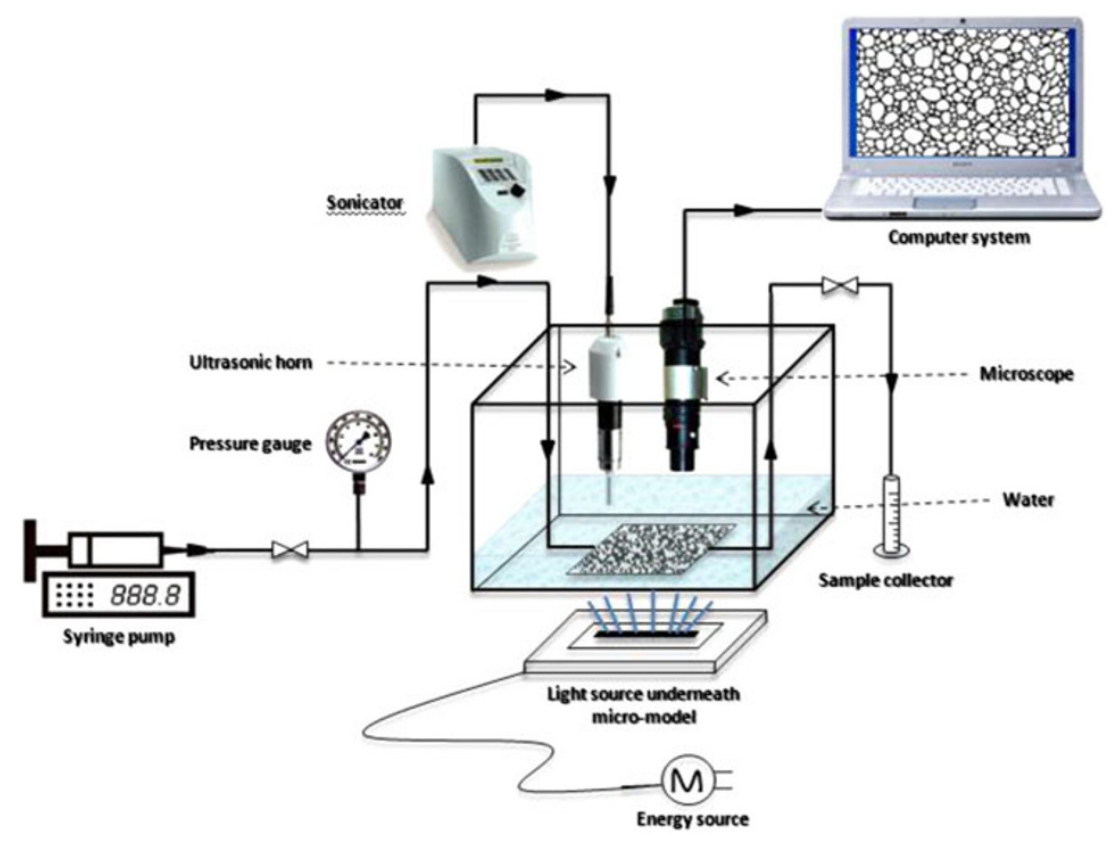

Fig. 4 Micro-model experimental setup 
Table 2 Properties of the oil

\begin{tabular}{lll}
\hline$T\left({ }^{\circ} \mathrm{C}\right)$ & Density $\left(\mathrm{g} / \mathrm{cm}^{3}\right)$ & Viscosity $(\mathrm{cp})$ \\
\hline 20 & 1.45 & 0.54 \\
\hline
\end{tabular}

Table 3 Experimental runs performed in this study

\begin{tabular}{llll}
\hline No. & Wettability & Power $(\mathrm{W})$ & Frequency $(\mathrm{kHz})$ \\
\hline 1 & Water wet & 350 & 20 \\
2 & Water wet & 350 & 40 \\
3 & Water wet & 350 & 68 \\
4 & Water wet & 50 & 40 \\
5 & Water wet & 180 & 40 \\
6 & Water wet & 350 & 40 \\
7 & Water wet & 500 & 40 \\
\hline
\end{tabular}

\section{Results}

Macro-model experiments

The recovery curves for water-wet experiment Nos. 1-3 with oil viscosity of $400 \mathrm{cp}$ are shown in Fig. 5. It is obvious that there is not any significant change in oil recovery rates due to ultrasonic waves. Therefore, the effect of ultrasonic waves on oil recovery for the experiments with heavy oil (400 cp) is trivial. Experiment Nos. 1 and 2 were performed again for repeatability. Figures 6 and 7 shows the error bars for these two experiments. Error bars showing the data variability and are applied on graphs in a measurement to show the error, or uncertainty. They are used to show that how precise a measurement is, or on the other hand, how far from the reported value the true (error free) value might be. As seen in Figs. 6 and 7, there is an early variation in oil recovery but ultimate recovery is approximately the same for the cases. The small variations at the beginning of the experiments could be ascribed to the alterations in the properties of cores since various cores were used in the experiments from the same geological block.

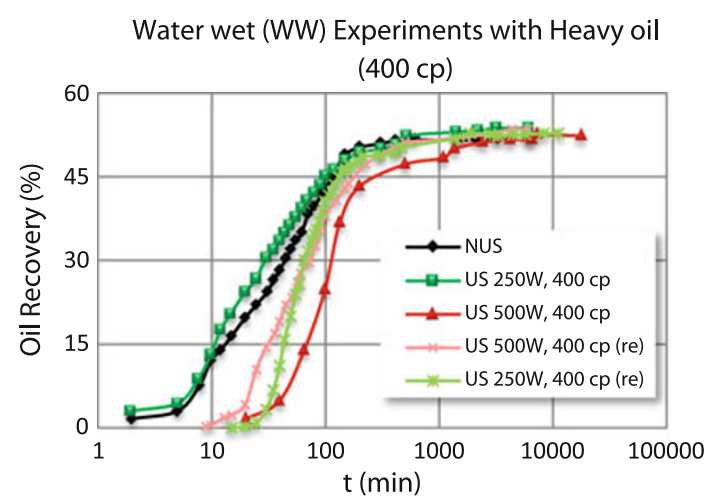

Fig. 5 Experiments 1-3, heavy oil $(400 \mathrm{cp})$ with ultrasound frequency of $25 \mathrm{kHz}$

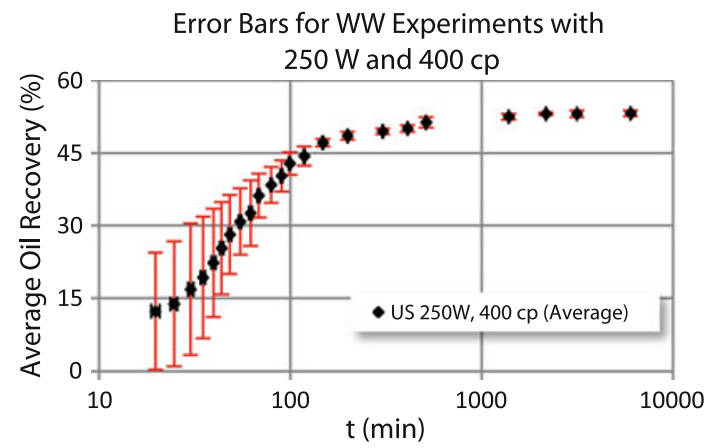

Fig. 6 Error bars for experiment No. 2, heavy oil (400 cp) with ultrasound power $250 \mathrm{kHz}$

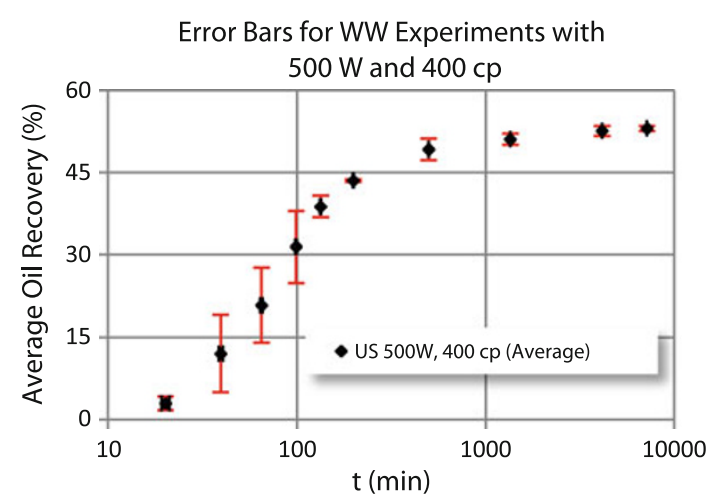

Fig. 7 Error bars for experiment No. 1, heavy oil $(400 \mathrm{cp})$ with ultrasound power of $500 \mathrm{~W}$

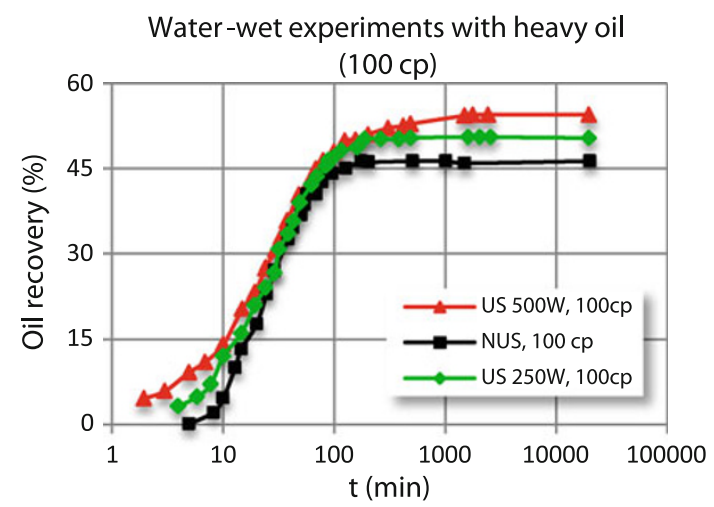

Fig. 8 Experiments 4-6, heavy oil $(100 \mathrm{cp})$ with ultrasound frequency of $25 \mathrm{kHz}$

The recovery curves for water-wet experiment Nos. 4-6 with oil viscosity of $100 \mathrm{cp}$ are shown in Fig. 8. It was observed that the ultimate oil recovery increased with increasing the power of the ultrasonic generator. Apparently, there are some possible mechanisms of oil recovery except the one explained in "Introduction" part control the process in these water-wet experiments, as following: (a) changing in interfacial tension (IFT) and wettability that 
were also mentioned before by Hamida and Babadagli (2007), (b) coalescence of oil droplets by Bjerknes forces that was also observed by Bjerknes (1906), (c) peristaltic movement of brine after applying ultrasound, which was mentioned before by Ganiev et al. (1989). The recovery curves for water-wet experiment Nos. 7-9 with oil viscosity of $15 \mathrm{cp}$ are shown in Fig. 9. As seen, the ultimate oil recovery increased under ultrasound, especially for the tests with power of $500 \mathrm{~W}$. The recovery curves for oil-wet experiment Nos. 10-13 with oil viscosity of 100 and $15 \mathrm{cp}$ are shown in Fig. 10. As seen, the ultrasound has a very considerable effect on oil mobilization and oil recovery rate. In these series of experiments, the ultimate recovery increased more than $100 \%$ by ultrasound compared to water-wet cases. The oil recovery mechanisms that may be responsible in these experiments except the one described in Introduction part are IFT changes and peristaltic movement of brine inside the porous media. In oil-wet cases, aqueous phase has more affinity to flow and lesser tendencies to adhere to wall surface of pores or throats.

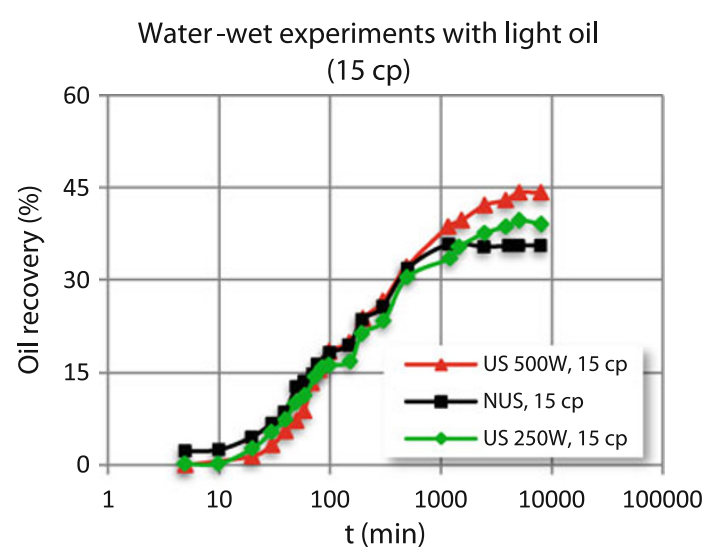

Fig. 9 Experiments 7-9, light oil (15 cp) with ultrasound frequency of $25 \mathrm{kHz}$

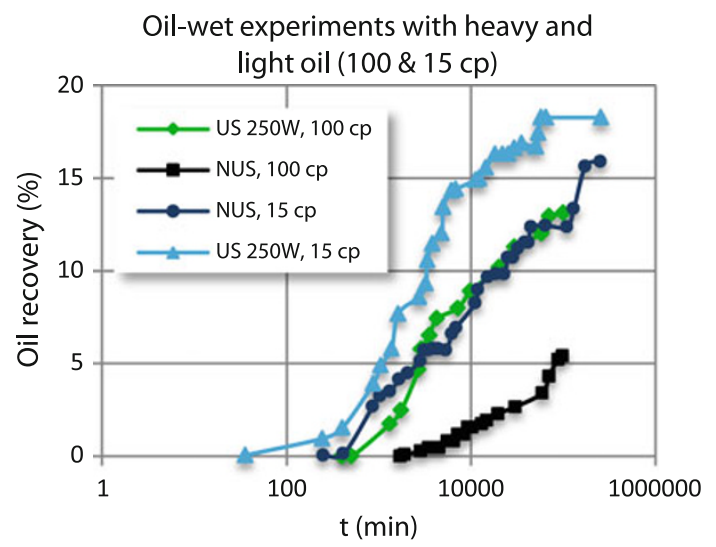

Fig. 10 Experiments 10-13, heavy and light oil (100 and $15 \mathrm{cp}$ ) with ultrasound frequency of $25 \mathrm{kHz}$
Therefore, peristaltic movement of aqueous phase may smooth the progress of movement of oil through porous media. The coalescence of oil droplet by Bjerknes forces is not an effective mechanism for oil-wet cases because in oil-wet cases the ganglion form of oil droplets does not exist. In addition, it can be seen that the effect of ultrasonic waves on oil recovery is more significant in oil-wet cases with viscosities of $15 \mathrm{cp}$.

The recovery curves for water-wet experiment Nos. 14-17 with oil viscosity of 100 and $15 \mathrm{cp}$, low ultrasound power $(1 \mathrm{~W})$ and ultrasound frequencies of 25 and $40 \mathrm{kHz}$ are shown in Fig. 11. These experiments were conducted to show the effect of frequency on oil recovery. The results show that for $40 \mathrm{kHz}$ frequencies the recovery rate increases but ultimate recovery for all four tests (40 and $25 \mathrm{kHz}$ ) was the same. For repeatability, the experiment Nos. 15 and 17 were repeated. These experiments are shown by (re) in the Fig. 11. Figures 12 and 13 show the error bars for these two experiments. The repeated test No. 17 demonstrates almost the same recovery trend; however, its final recovery shows a few percent alteration compare to the first experiment No. 17. This alteration could be due to a variation in core properties or conditions of the experiments. For experiment No. 15, the repeated curve of recovery has a close match with its first one.

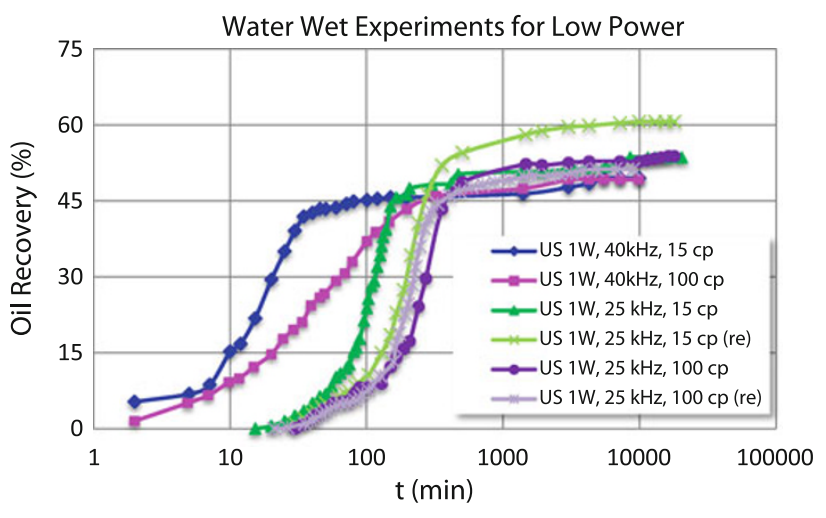

Fig. 11 Experiments 14-17, heavy and light oil (100 and $15 \mathrm{cp}$ ) with ultrasound frequency of 25 and $40 \mathrm{kHz}$

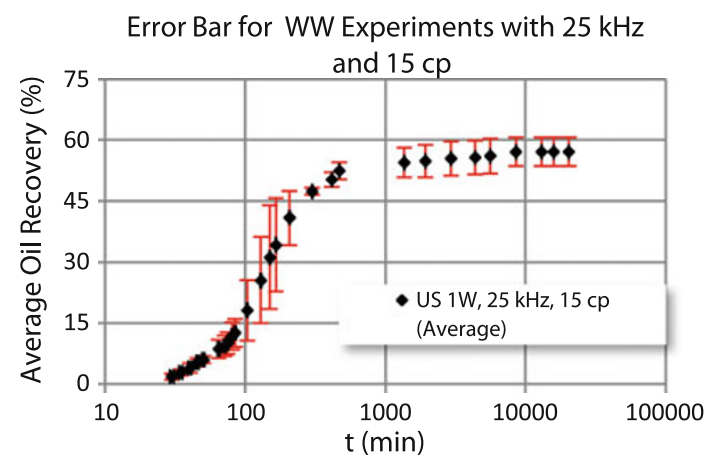

Fig. 12 Error bars for experiment No. 17, light oil 
Error Bar for WW Experiments with $25 \mathrm{kHz}$

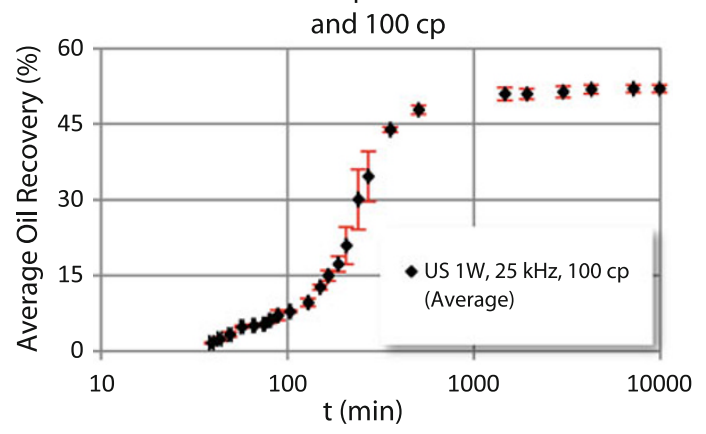

Fig. 13 Error bars for experiment No. 15, (15 cp) with ultrasound power of $1 \mathrm{~W}$, heavy oil $(100 \mathrm{cp})$ with ultrasound power of $1 \mathrm{~W}$

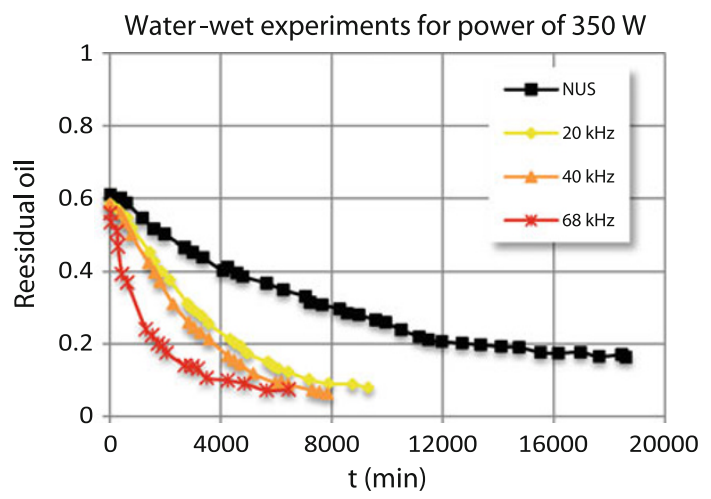

Fig. 14 Residual oil versus time for experiments done at different ultrasound frequencies and constant power

\section{Micro-model experiments}

The result of water-wet experiments 1-3 at an ultrasound power of $350 \mathrm{~W}$ and different frequencies of 20, 40, and $68 \mathrm{kHz}$ are shown in Fig. 14. This figure shows the residual oil saturation as a function of time. The purpose of doing these experiments was to understand the effect of ultrasonic frequency on oil mobilization. As seen, it is obvious that the oil mobilization in porous media is dependant to ultrasound frequency and residual oil decreased much faster than for the case without vibration (NUS).

For the repeatability, three experiments of water flood were conducted in the absence of ultrasound (Fig. 15). As seen, the general trends for all the three experiments are analogous, particularly at the first displacement period but they deviate as they come up to the end of process. Figure 16 shows the error bars for the experiments. These deviations are possibly due to statistical variations related to the size of the lattice. Specifically, the preliminary pathways of percolation control the saturation of the residual oil. In addition to these experiments, three other experiments were performed and repeated at ultrasound frequency of $68 \mathrm{kHz}$ and power of $350 \mathrm{~W}$ (experiment No. 3) (Fig. 17).

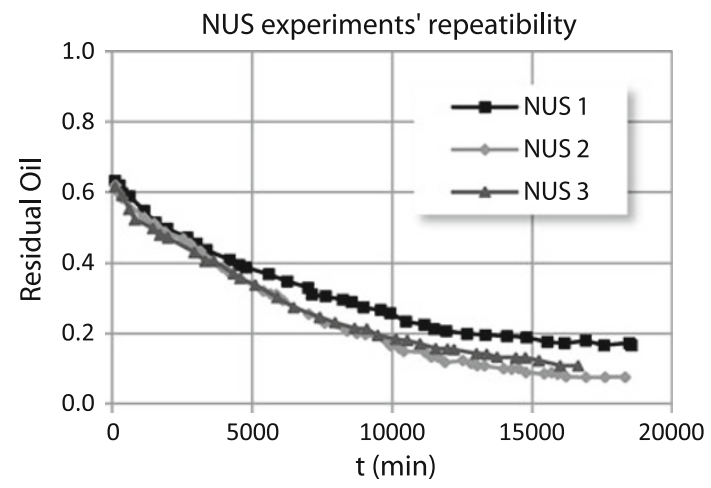

Fig. 15 Residual oil versus time for three NUS repeated experiments

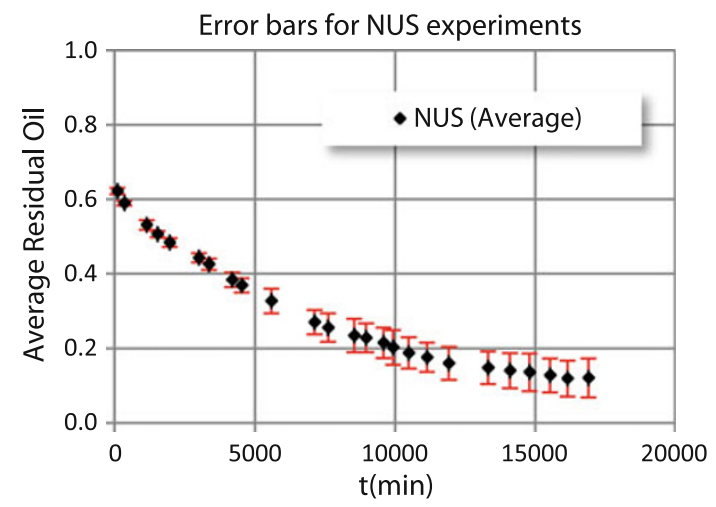

Fig. 16 Error bars for NUS experiments

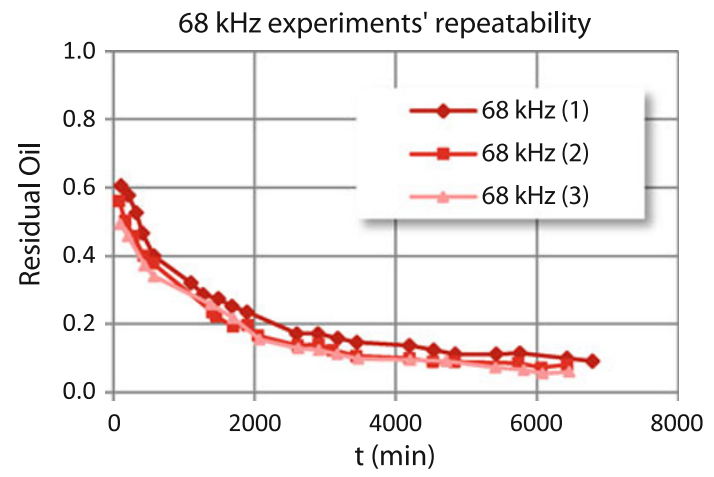

Fig. 17 Residual oil versus time for repeated experiments done with ultrasound frequency of $68 \mathrm{kHz}$ and power of $350 \mathrm{~W}$

Figure 18 shows the error bars for the experiments. As seen, the repeatability for the tests is very good and much better than obtained for the case of NUS. Because the force generated by ultrasound exerted on all sections of the micromodel at the same time and without inequity, the probability of growing pathways of percolation which bypass great flow cell portions (as a result of that causes larger variations from one experiment to another) is reduced. 


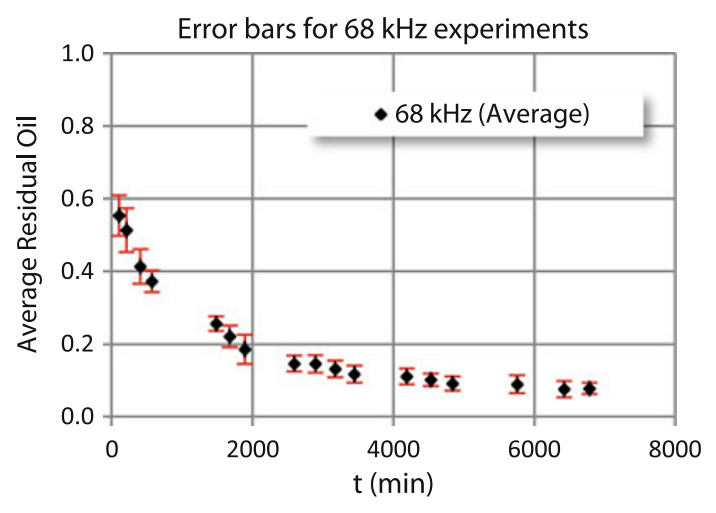

Fig. 18 Error bars for experiments No. 3 with experiments done with ultrasound frequency of ultrasound frequency of $68 \mathrm{kHz}$ and power of $350 \mathrm{~W}$

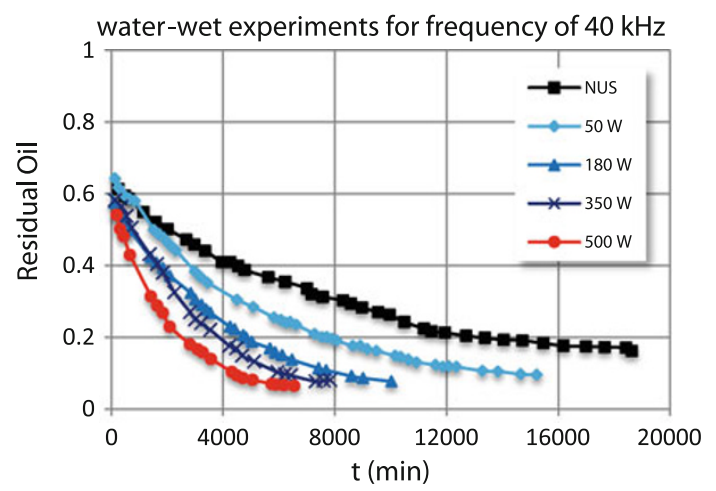

Fig. 19 Residual oil versus time for experiments done at different ultrasound power and constant frequency

The result of water-wet experiments 4-7 at an ultrasound frequency of $40 \mathrm{kHz}$ and different powers of 50 , 180, 350 and $500 \mathrm{~W}$ are shown in Fig. 19. This figure shows the residual oil saturation as a function of time. As seen, the mobilization of oil inside porous media is also dependant to ultrasound power. Therefore, for greater powers the residual oil saturation decreased much faster than for the case without vibration (NUS). With respect to Figs. 14 and 19, it was concluded that the predictions summarized in the "Introduction" part, are validated by conducting these series of experiments.

\section{Conclusions}

In this study, the macro-model and 2D glass micro-model experiments were performed to prove the predictions of a mechanism of oil mobilization in porous media under ultrasonic waves. The result of the macro-model experiments showed that:
- the oil recovery increases under ultrasonic waves.

- The effect of ultrasound on oil recovery in a medium with high oil viscosity is less than the cases with lower viscosities.

- The effect of ultrasonic waves on oil recovery is more significant in oil-wet cases especially with lower viscosities.

- The recovery rate for higher frequency is more significant than lower frequency. Nevertheless, the ultimate recovery was almost the same for two cases.

The result of the micro-model experiments showed that:

- when the frequency increases from 20 to $68 \mathrm{kHz}$ for constant ultrasound power, the oil droplet move faster in the water flood experiments.

- When the ultrasound power increases from 50 to $500 \mathrm{~W}$ for constant ultrasound frequency $(40 \mathrm{kHz})$, the oil droplet move faster in the water flood experiments. So, the residual oil saturation decreases.

In general, the findings of this study show that the rate of oil production is proportional to the ultrasound power and frequency.

Acknowledgments The authors appreciate the Faculty of Petroleum and Renewable Energy Engineering at University Technology Malaysia in Johor Bahru, Malaysia for providing laboratory facilities in completing this work.

Open Access This article is distributed under the terms of the Creative Commons Attribution License which permits any use, distribution and reproduction in any medium, provided the original author(s) and source are credited.

\section{References}

Amro MM, Al-Mobarky A, Al-Homadhi ES (2007) Improved oil recovery by application of ultrasound waves to waterflooding. In: SPE 105370, presented at the 15th SPE Middle East Oil and Gas Show and Conference held in Bahrain International Exhibition Centre, Kingdom of Bahrain, 11-14 March

Beresnev IA, Vigil D, Li W (2005) Elastic waves push organic fluids from reservoir rock. Geophys Res Lett 32:L13303. doi: $10.1029 / 2005 \mathrm{~g} 1023123$

Bjerknes VF (1906) Fields of Force. Columbia University Press, New York

Chen WI (1969) Influence of ultrasonic energy upon the rate of flow of liquids through porous media. Chemical Engineering. $\mathrm{PhD}$ thesis, West Virginia University, p 141

Duhon RD, Campbell JM (1965) SPE 1316 - the effect of ultrasonic energy on flow through porous media. In: Second Annual Eastern Regional Meeting of SPE/AIME, Charleston, WV

Fairbanks HV, Chen WI (1971) Ultrasonic acceleration of liquid flow through porous media. Chem Eng Prog Symp Ser 67:108

Ganiev RF, Ukrainskii LE, Frolov KV (1989) Wave mechanism for the acceleration of a liquid flowing in capillaries and porous media. Sov Phys Doklady 34:267-283 
Hamida T, Babadagli T (2007) Fluid-fluid interaction during miscible and immiscible displacement under ultrasonic waves. Eur Phys J B 60:447-462. doi:10.1140/epjb/e2008-00005-5

Kostrov S, Wooden BO (2001) Mechanisms, flied suitability, and case studies for enhancement of oil recovery and production using in situ seismic stimulation. international conference elastic wave effect on fluid in the porous media. In: International symposium on nonlinear acoustics, Moscow
Simkin EM (1993) A possible mechanism of vibroseismic action on an oil-bearing bed. J Eng Phys Thermophys 64(4):355-359

Simkin EM, Surguchev ML (1991) Advanced vibroseismic technique for water flooded reservoir stimulation, mechanism and field tests results. In: The 6th European IOR Symposium, Stavanger, Norway 